Théologiques

Théologiques

\title{
Aux frontières du silence : Exploration du dialogue interreligieux monastique
}

\section{Fabrice Blée}

Volume 7, numéro 2, automne 1999

Silence !

URI : https://id.erudit.org/iderudit/005000ar

DOI : https://doi.org/10.7202/005000ar

Aller au sommaire du numéro

\section{Éditeur(s)}

Faculté de théologie de l'Université de Montréal

\section{ISSN}

1188-7109 (imprimé)

1492-1413 (numérique)

Découvrir la revue

\section{Citer cet article}

Blée, F. (1999). Aux frontières du silence : Exploration du dialogue interreligieux monastique. Théologiques, 7(2), 79-94.

https://doi.org/10.7202/005000ar
Résumé de l'article

Le silence comme lieu de communion dans l'expérience du

dialogueinterreligieux monastique. L'article veut articuler la signification et la placedu silence dans la dynamique dialogale qui se dégage de la rencontre entredes moines chrétiens et des moines non-chrétiens. d'utilisation que vous pouvez consulter en ligne. 


\title{
Aux frontières du silence Exploration du dialogue interreligieux monastique
}

\author{
Fabrice BLÉE \\ Faculté de théologie \\ U. de M.
}

\section{Introduction}

Parmi les expériences interreligieuses engagées dans les années post-conciliaires, celles des 0 rdres contemplatifs sont au nombre des plus fructueuses et des plus avancées. Un dialogue avec les renonçants principalement bouddhistes et hindous a été entrepris sous les auspices des autorités bénédictines et cisterciennes, de concert avec le Vatican, dès la fin des années 1970 avec la création de deux commissions, I'une en Europe(Dialogue Interreligieux M onastique) et l'autre en A mérique du Nord ( $\mathrm{N}$ orth A merican Board for East-West Dialogue ${ }^{1}$ ). L e dialogue des moines ${ }^{2}$ est prometteur car il ne se résume ni à un simple échange verbal ni à une action commune contre l'injustice, mais repose avant tout sur le partage des expériences de prière, de contemplation et de foi des partenaires du dialogue. À ce niveau d'échange, il appert que les partenaires «s'entendent d'emblée merveilleusement $»{ }^{3}$ Ce type de dialogue, aussi appelé de "l'expérience religieuse », ${ }^{4}$ se situe au sein de l'Église catholique à la croisée des mouvements contemplatif et dialogal, del'expérience non-

1. Le N.A.B.E.W.D. deviendra le Monastic Interreligious Dialogue (M .I.D.) en 1993.

2. N ous emploierons le terme de « moines » pour désigner les moines et les moniales, afin de ne pas alourdir le texte.

3. P.-F. DE BÉTHUNE, «Le Silence, chemin de dialogue. Réflexions sur l'expérience des moines en dialogue », Chemins de dialogue6 (oct. 1995), p. 201. 4. Voir le document pontifical «Attitude de l'Église catholique devant les croyants des autres religions. Réflexions et orientations concernant ledialogueet la mission », Bulletin. Secretarius pro non Christianis (Pentecôte 1984), p. 19. 
duelle du divin et du rapport intime à l'altérité religieuse, du silence et de la parole. $\mathrm{N}$ otre étude du dialogue interreligieux monastique menée depuis $1992^{5}$, révèle que tout dialogue effectué en profondeur, de cor ad cor, est nécessairement marqué du sceau de cette contradiction apparente dans les termes. La communication, en effet, ne peut devenir communion sans un rapport intime au silence. Le Secrétaire général du dialogue interreligieux monastique, P.-F. de Béthune, écrit à ce titre que la « rencontre la plus décisive se vit [...] dans l'expérience commune de l'indicible, au delà des mots $\gg .{ }^{6}$ Avant lui, un des pionniers en ce domaine, Henri Le Saux, affirmait qu'un dialogue sans silence est nécessairement suspect. ${ }^{7}$ O n comprend alors pourquoi le dialogue des moines est désigné aussi par l'expression : « dialogue de silence $\gg .{ }^{8} \mathrm{D}$ ans cet article, nous tenterons de comprendre et d'articuler la signification et la place du silence dans la dynamique dialogale qui se dégage de la rencontre entre les monachismes chrétien et nonchrétien. Le dialogue des moines est un dialogue de silence pour essentiellement deux raisons : d'un côté, il se développe sans faire de bruit en vertu de son caractère proprement monastique ; del'autre, il prend corps au dedans du moine dans l'intimité de sa démarche spirituelle. Par conséquent, notre regard sur ce dialogue peu ordinaire sera double: nous le considérons d'abord d'un point de vue contextuel, attentif au silence qui l'entoure, puis nous nous proposons de l'aborder d'un point de vue expérientiel, en nous arrêtant sur le fait qu'il se situe au cœur du silence.

\section{Le silence autour du dialogue}

On reçoit encore peu d'échos du dialogue des moines autant dans les écrits théologiques que dans les textes officiels de l'Église, malgré une expérience dans le domaine accumulée depuis une trentaine

5. Voir F. BLÉE, LeDialogue interreligieux monastique. L'expériencenordaméricaine. Histoire et analyse [thèse de doctorat], $M$ ontréal, Université de M ontréal, 1999, 496p.

6. P.-F. DE BÉTHUNE, Ibid., p. 206.

7. J. WISEMAN, «Initiating Regional and Local Dialogue», Bulletin. N orth American Board for East-West D ialogue 42 (1991), p. 10.

8. Voir Bulletin of M onastic Interreligious Dialogue 48 (1993), p.15 et P.F. DE BÉTH UNE, « Le Silence, chemin de dialogue. Réflexions sur l'expérience des moines en dialogue », Chemins de dialogue 6 (octobre 1995), p. 201-207. 
d'années, un fait d'autant plus étonnant que ce dialogue a été vivement encouragé par Paul VI et J ean Paul II et qu'il est un exemple de dialogue réussi. La discrétion qui l'entoure s'explique par le fait que le silence est le lieu privilégié sur lequel il s'enracine et s'articule. II faut en outre se rendre compte qu'il est aussi un travail de fond, en ce sens exigeant et de longue haleine, qui vise ni plus ni moins la compréhension mutuelle entre les partenaires engagés.

\subsection{Le silence comme lieu de dialogue}

Si l'on se reporteà l'histoire du dialogue des moines et à son caractère proprement monastique, il appert que ce dialogue se développe sur la base d'un rapport silencieux à l'autre plutôt que verbal. Les moines de saint Benoît furent particulièrement sensibilisés à la nécessité d'engager un dialogue avec les moines d'autres religions au congrès panasiatique de Bangkok en 1968. Ce congrès s'inscrit dans le nouvel effort missionnaire de la famille bénédictine entrepris depuis le début des années 1960 dans les pays dits de mission. De tous les continents, I'A sie s'est très vite démarquée en défiant à maints égards les moines-missionnaires. Un de ces défis consistait à briser l'image d'un christianisme « colonial » essentiellement activiste et donc très peu spirituel. En Inde, P. D 'souza, évêque de Bangalore, constatait avec regret en 1973 que pour « les hommes de ce pays, nous avons été un signe de l'amour du prochain, mais nous n'avons pas réussi à être signes de la présence de Dieu en nous et autour de nous ». ${ }^{9}$ Pour s'implanter en A sie, il fallait donc miser sur deux choses : premièrement, soutenir que l'essence de la vie monastique est la recherche de la contemplation, de la sagesse, de la connaissance de D ieu et, deuxièmement, défendre l'idée de la désoccidentalisation du monachisme chrétien, afin que celui-ci soit en mesure, non pas de s'adapter aux cultures asiatiques, mais d'en devenir une dimension constitutive.

Ici, le dialogue en profondeur avec les spiritualités orientales se révéla incontournable. L'hindouisme et le bouddhisme en particulier sont à l'origine de comportements éthiques et religieux profondément ancrés dans bien des aires culturelles d'A sie comme le silence, le renoncement, la pauvreté, l'intériorité ou encore l'expérience de la non-dua-

9. J. LECLERCQ, N ouvelle page d'histoire monastique, histoire de I'A.I.M . 1960-1985, Paris, Publication de l'A.I.M ., 1986, 224p. 
lité. D'où l'appel, en tant que chrétien, à se convertir en quelque sorte aux valeurs essentielles de l'hindouisme et du bouddhisme, un travail tout désigné pour les moines de saint Benoît pour qui la vie se centre autour de la contemplation et du silence. Conscient de cela, le Cardinal S. Pignedoli fit parvenir une lettre à l'A bbé Primat des Bénédictins en 1974 pour l'encourager à poursuivre le dialogue en O rient, convaincu que le moine représente au mieux I'homo religiosus, point d'attraction et de référence pour les chrétiens et les non-chrétiens.

D ès ses débuts, le dialogue intermonastique s'affiche comme étant un dialogue de silence, parce que hindous et bouddhistes sont naturellement peu enclins à participer à de longs débats théologiques ${ }^{10}$ et qu'il est plus aisé de les rencontrer au niveau de la prière et du silence qu'à celui de la pensée et des doctrines ${ }^{11}$. $D^{\prime}$ 'où cette préoccupation d'entrer le plus concrètement possible dans la recherche spirituelle de I'autre ; ce qui est particulièrement vrai dans le dialogue avec les bouddhistes qui, comme le remarque très justement P.-F. de Béthune sont toujours discrets, « plus soucieux de la justesse de la question que de la pertinence de la réponse $»{ }^{12}$ A joutons à cela que le silence autour des rencontres interreligieuses se trouve renforcé compte tenu que celles-ci se déroulent dans les monastères tantôt chrétiens tantôt bouddhiques, souvent à huis clos, en marge des activités laïques. La fameuse rencontre de G ethsémani ${ }^{13}$, par exemple, qui s'est tenue du 22 au 27 juillet 1996, n'était pas ouverte au public ; un certain nombre de médias ont toutefois été autorisés à immortaliser l'événement. II faut en réalité se garder d'y voir la promotion d'une élite interreligieuse. Les moines en dialogue n'entretiennent pas le secret ni même n'encouragent la discrétion au sujet de leurs objectifs et de leurs

10. D. Steindl-R ast rapporte: «Dialogue is an aspect of that sharing. But sharing is more than mere dialogue. In dialogue the emphasis falls on word and speech. This emphasis is a typically Christian preoccupation. Why should it be imposed on Buddhists? Silence, too, can be shared. »; dans «A Shift in Buddhist-Christian Dialogue », Bulletin. N orth American Board for EastWest Dialogue 30 (1987), p. 13.

11. Voir Bulletin. North American Board for East-West Dialogue 41 (1991), p. 5.

12. P.-F. DE BÉTHUne, Ibid., p. 204.

13. Voir F. BLÉE, «Une Rencontre historique», Présence magazine (N ovembre 1996), p. 5. 
activités ${ }^{14}$, mais se préoccupent par contre de préserver le caractère proprement monastique de leurs échanges.

\subsection{Un dialogue de fond}

Le dialogue interreligieux monastique se développe sans éclat compte tenu aussi du fait qu'il constitue un travail de fond, in vivo, par opposition aux rencontres in vitro d'envergure nationale ou internationale largement médiatisées, comme la prière pour la paix d'A ssise (1986) ou le Parlement des religions à Chicago (1993), et qui sont le plus souvent sans lien véritable avec les réalités socio-politicoéconomiques des partenaires engagés. À l'inverse, les échanges intermonastiques, plus modestes, sont aussi plus exigeants car ils visent la compréhension mutuelle entre les partenaires et non, en premier lieu, à informer, à convaincre et encore moins à convertir.

$M$ ais les moines en dialogue nous enseignent que se comprendre les uns les autres n'est possible que dans un contact prolongé au rythme de la vie quotidienne dans une prise en compte des réalités concrètes de chacun. Songeons à leur activité principale qui consiste en un programme d'hospitalité mis sur pied tant en Europe (1979) qu'aux États-U nis (1982) et qui permet, d'un côté, à des moines chrétiens de vivre plusieurs semaines dans des monastères bouddhiques au Japon, au Tibet et en Inde et, de l'autre, à des moines bouddhistes de séjourner plusieurs mois dans des abbayes occidentales. De ces échanges, il ressort que dans les premiers temps les moines se sont confrontés à l'impossibilité de s'engager dans un dialogue proprement dit, faute de connaissances adéquates du bouddhisme et de son monachisme. $M$ ais ils étaient cependant en mesure de se rendre l'hospitalité, première étape reconnue par tous sur la voie d'un dialogue profond. L'hospitalité est le prélude obligéà un dialogue plus affirmé ; elle offre un environnement propice à l'éclosion d'une amitié fraternelle entre les participants. La fraternité qui se construit avec patience et fidélité au fur et à mesure des rencontres est la pierre angulaire du dialogue intermonastique. Elle permet de surmonter un choc culturel

14. P. C off nous a écrit dans sa lettre du 3 septembre 1996 : «It was never intended that MID become a public phenomenon, but neither is she to be hidden a bushel. » 
et religieux inévitable, une incompréhension face à des idées et des comportements inconnus, voire déroutants.

$M$ ais il vient un temps, où, fort de leur relation amicale, les parties concernées sont prêtes à engager un dialogue à partir des concepts qui régissent la cohérence de leurs univers religieux respectifs. Du côté américain, ce temps est venu de façon évidente six ans après le premier échange de 1982 avec des moines tibétains. N ous sommes donc en face d'un travail de longue haleine, dont les premiers résultats sont difficilement perceptibles dans l'immédiat. A insi le succès incontesté de la rencontre de G ethsémani de 1996 repose sur quinze ans d'hospitalité et d'efforts pour nourrir des amitiés, établir la confiance réciproque et se comprendre l'un l'autre. II est en réalité le résultat « de toute une évolution intérieure et même d'un profond changement des mentalités grâce auxquelles I'O rdre monastique pouvait enfin se reconnaître dans les intuitions des quel ques précurseurs $» .{ }^{15}$ Finalement, le silencequi entoure le dialogue des moines est en quelque sorte à la mesure des tranformations profondes et peu apparentes qu'il suscite chez les partenaires.

\section{Le dialogue au cœur du silence}

La notion de silence rattachée au dialogue des moines se rapporte aussi a une dimension plus intérieure de la rencontre avec l'altérité religieuse. O n parle dans ce cas d' « expérience de silence». Cette expression, tirée du document "Contemplation et dialogue interreligieux » (1993), touche au caractère spécifique du dialogue intermonastique. Elle désigne ce comportement nouveau et particulier pour un chrétien qui consiste à adopter une pratique de méditation silencieuse ou de " silence contemplatif » ${ }^{16}$ issue d'une autre religion. Songeons par exemple à zazen, vipassanâ, rD zogs chen et à certains yoga. La pratique de telles méditations peut engager le moine sur la voie d'un dialogue, quel'on qualifie, à la suite de R. Panikkar, d'intrareligieux, c'est-à-dire qui se développe au-dedans de la personne. Celle-ci se retrouve aux prises avec deux visions du monde, deux

15. M anuscrit en français de P.-F. de Béthune de la préface pour les actes de la rencontre de $\mathrm{G}$ ethsémani, p. 6.

16. «Contemplation et dialogue interreligieux. Repères et perspectives puisés dans l'expérience des moines », Bulletin. Secretarius pro non Christianis 84 (1993-X X VIII/3), p. 99. 
façons de penser et de sentir, qui ne peuvent s'assimiler l'une l'autre, dès l'instant où I'on respecte le caractère spécifique et universel de ces pratiques et la cohérence religieuse dont elles sont issues et qu'elles véhiculent par ailleurs.

O n comprend alors très bien que le dialogue ne correspond pas ici à une activité secondaire que l'on peut quitter et reprendre à volonté ; il s'apparente davantage à une voie spirituelle à part entière, comportant un risque existentiel, du fait qu'il devient partie intégrante de la personne ou encore une nouvelle façon d'être au monde. C'est un dialogue qui se développe dans le silence des profondeurs del'être et dont le fondement est à rechercher dans cette dimension essentielle de I'humain, que H. Le Saux appelle le « cœur » (guha), et qui renvoie au-delà des mots, au mystère de Dieu qui est aussi mystère d'amour. « M y dear brothers, a déclaré T. M erton à Calcutta en 1968, we are already one. But we imagine that we are not. And what we have to recover is our original unity. What we have to be is what we are ${ }^{17}$ Le dialogue des moines repose sur l'arrière-fond de cette unité originelle, au-delà des mots, si bien que tout échange verbal, toute parole prononcée entre les partenaires se trouve enrichi d'un silence empli d'une présence à la fois une et multiple.

$M$ ais tentons à présent d'articuler plus à fond la place du silence et sa signification dans l'expérience intrareligieuse des moines. Pour cela, reportons-nous aux trois grandes dimensions de cette expérience que nous avons dégagées dans le cadre de notre recherche sur le $M$ onastic Interreligious Dialogue (M .I.D.). Ces dimensions sont I'engagement vis-à-vis du partenaire de dialogue, I'enracinement dans sa propre tradition religieuse et le cheminement intérieur qui découle de la rencontre des deux premières dimensions.

\subsection{Le silence de l'engagement}

S'adonner en tant que chrétien à une pratique de méditation bouddhique ou hindoue n'est jamais anodin, et ce, quelle que soit la place que I'on veut bien lui assigner dans sa propre démarche spirituelle. P.-F. de Béthune rappelle à juste titre que l'adoption d'une de ces méthodes revient en définitive à accueillir des personnes. On s'ouvre en effet à

17. Bulletin. N orth American Board for East-West D ialogue 18 (1983), p. 6. 
« une tradition élaborée par des générations de chercheurs de l'A bsolu ${ }^{18}{ }^{18}$ Dans la logique de ces propos, il apparaît inévitable que la pratique du zen ou du yoga, qui déboucheà la fois sur une spiritualité du corps et la non-dualité del'esprit, contribue au fait quele moine chrétien en vienne à intérioriser l'altérité religieuse dans tout son être et en premier lieu dans sa corporéité. II ne s'agit pas là d'un engagement ponctuel et sans conséquence, mais bien total et irréversible; et cela l'est d'autant plus que le moine fait sien la vérité universelle de l'autre. Le dialogue intrareligieux pousse donc le moineà vivre l'hospitalité jusqu'au bout. Si le disciple de saint Benoît trouve important de recevoir l'étranger, il oubliesouvent del'accueillir avec la dimension religieuse qui lui est propre et dont il ne peut d'ailleurs se départir d'aucune façon.

Le moine en dialogue va, quant à lui, jusqu'à recevoir l'autre avec ses convictions religieuses les plus intimes dans l'espoir de partager ce que tous deux ont de plus précieux ${ }^{19}$ dans la perspective de découvrir ensemble la Vérité une et multiple et d'agir en accord avec elle. En d'autres mots, I'hôte est un envoyé de Dieu aussi par son appartenance religieuse. Les Pères du dialogue intermonastique, T. M erton, H. Le Saux et B. Griffiths, ont ouvert cette voie de I'hospitalité interreligieuse qui est aussi réciproque et intrareligieuse. Ils ne se contentent pas en effet d'accueillir l'autre croyant sous leur toit et d'être accueillis sous le sien, mais le reçoivent aussi dans leur cœur. $D$ 'où cette composante spécifique du dialogue des moines qui consiste à s'offrir mutuellement un espace en soi. On retrouve ici l'idée de silence ; l'espace et le silence étant deux réalités connexes propres à l'ouverture à l'autre. Cela transparaît, nous semble-t-il, dans le langage même del'hospitalité qui est moins del'ordre del'échange verbal que du geste et de la présence silencieuse. Un sourire, un regard, l'humour et le don de soi ont souvent beaucoup plus de poids qu'un discours bien soigné dans le rapprochement de deux êtres que séparent la religion, l'origine ethnique ou encore la langue.

18. «Contemplation et dialogue interreligieux. Repères et perspectives puisés dans l'expérience des moines », Bulletin. Secretarius pro non Christianis 84 (1993-XX VIII/3), p. 117.

19. Voir H. LE SAUX, «The Depth-Dimension of Religious Dialogue», Bulletin. N orth A merican Board for East-West D ialogue 13 (1982), p. 5. 
$M$ ais nous comprenons bien que si le silence est un terrain propice à la rencontre interreligieuse, puisqu'il permet de dépasser les impasses doctrinales et théologiques, il ne se présente pas comme un moyen de fuir la confrontation ou de taire les spécificités de chacun en vue d'une entente à tout prix. Le silence ne traduit pas ici une absence de convictions. Lorsque les moines partagent des moments de silence, que ce soit lors d'une méditation, d'une prière ou d'une activité quelconque de la journée, ils ne se désengagent pas les uns par rapport aux autres. Le silence est au contraire synonyme d'engagement du fait qu'il renvoie avant tout à l'action d'écouter. A ucune compréhension mutuelle, aucune hospitalité n'est possible sans l'écoute préalable de l'autre. L'écoute à laquelle nous faisons référence ici n'est pas passive et ne cherche ni la confirmation de mes propres idées ni la conversion par des moyens détournés de mon vis-à-vis. À ce titre, l'écoute mue par une intention séparée du fait même d'écouter ne serait que l'apparence silencieuse du bruit des peurs et des espoirs de celui-là même qui écoute.

Chez le moine en dialogue cette écoute désigne plutôt une prédisposition de l'esprit et du cœur, nécessaire au développement du dialogue qui consiste à se libérer des automatismes, des routines, des conventions, et aussi des idées préconçues que l'on se fait de l'autre, qui empêchent toute disponibilité à l'imprévu et à l'inédit. ${ }^{20}$ II s'agit d'appréhender chaque situation comme authentiquement nouvelle, c'est-à-dire refuser de contrôler sa propre destinée et accepter que le chemin se crée en marchant. II s'agit en outre de considérer l'autre non pas comme un objet, un être extérieur à soi, dont il faut se protéger, mais bien comme un sujet avec qui on est étroitement et fondamentalement lié en dépit des apparences. Vu sous cet angle, l'écoute est certainement un acte de foi et d'amour qui se rapporte à ce que P. Coff appelle, à la suite de S. Suzuki, le beginner's mind ${ }^{21}$ et David Steindl-R ast, le good poet. ${ }^{22} \mathrm{M}$ ais, encore une fois, développer une telle écoute exige de savoir faire silence. D'où cette condition apportée par T. M erton : le dialogue monastique « doit être réservé

20. Voir T. Merton (AJ,313) et R. PAnIKkar (DIA,99).

21. Voir P. COFF, «The Inner Journey : R eflections on the Awakening of $M$ ind and $\mathrm{H}$ eart in Buddhism and Christianity », Buddhist-Christian Studies 11 (1991), p. 176.

22. Voir D. StEIN DL-RAST et R. AITKEN, The G round We Share. Everyday Practice, Buddhist and Christian, Liguori, Triumph Books, 1994, p. 186. 
à ceux qui ont été formés par des années de silence et une longue habitude de la méditation $\gg .23$

\subsection{Le silence de l'enracinement}

L'accueil en soi de la vérité universelle de l'autre croyant ne com-

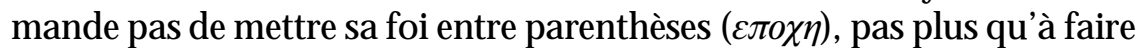
des compromis sur des points dethéologie ou de doctrines. L'expérience démontre au contraire que la rencontre intime avec les spiritualités oriental es pousse le moine à s'enraciner davantage dans sa tradition, à la connaître et à la vivre plus en profondeur. L'enracinement s'impose comme un préalable, une préparation nécessaire au dialogue. Songeons ici à T. M erton pour qui ce dernier « doit être réservé à ceux qui sont entrés très sérieusement dans leur propre tradition monastique et sont en contact authentique avec le passé de leur propre communauté religieuse $»{ }^{24}$ L'enracinement se présente aussi comme la conséquence d'une confrontation avec la différence religieuse ou, pour le dire dans les mots du Dalaï Lama, d'une « compétition constructive » ${ }^{25}$, où chacun tente de donner le meilleur de lui-même, en mettant de son mieux en pratique les enseignements de sa propre religion. Une des premières choses que l'hindou demande au chrétien est souvent de savoir si ce dernier a fait l'expérience, sous-entendue du divin en soi (advaita). Cette question est souvent embarrassante, mais elle a le mérite de provoquer l'auto-critique chez celui qui la reçoit et de susciter dans bien des cas le désir d'un retour à une expérience contemplative de Dieu. Plus Iargement, I'O rient, I'Inde en particulier, possède ce don de provoquer spirituellement le moine chrétien en ce sens; plusieurs rapportent y avoir fait une expérience déroutante et révélatrice : expérience d'illumination pour T. $M$ erton ${ }^{26}$, de wholeness et de non-dualité (not-twoness) pour C. Tholens ${ }^{27}$, de

23. «Contemplation et dialogue interreligieux. Repères et perspectives puisés dans l'expérience des moines », Bulletin. Secretarius pro non Christianis 84 (1993-XXVIII/3), p. 107-108.

24. Ibid.

25. Dalaï LAMA, «Harmony, Dialogue and Meditation», Bulletin of M onastic I nterreligious D ialogue 57 (1997), p.13.

26. Voir T. MERTON, Thoughts on the East, N ew York, $\mathrm{N}$ ew Directions, 1995, p. 84.

27. Bulletin. N orth A merican Board for East-West Dialogue 5 (1979), p. 6. 
sacredness pour B. Griffiths ${ }^{28}$ ou encore d'intériorité pour P. Coff. ${ }^{29}$ II faut rappeler aussi que H. Le Saux entreprit sa " saga » au moment où il fit l'expérience de la présence silencieuse et transfigurée de $R$ amana $M$ aharshi ${ }^{30}$, un événement à la suite duquel le Bénédictin n'avait plus qu'une idée en tête : devenir un "Ramana chrétien $\gg .{ }^{31}$ L'expérience intrareligieuse incite en réalité les moines de saint Benoît à retrouver une spiritual ité beaucoup plus incarnée. Le document important de 1993 sur les moines en dialogue atteste que ceux-ci reconnaissent le rôle déterminant des méditations nonchrétiennes dans la pleine prise de conscience de leur corps, de leur souffle et de leur psychisme. ${ }^{32} \mathrm{D}$ 'où la réconciliation dans certains cas avec cet aspect de la tradition mystique chrétienne qu'est l'expérience subjective du divin à la fois transcendant et immanent. Si Dieu est inaccessible, au-delà de tout système religieux, son royaume est pourtant en chacun de nous, comme l'affirmait Jésus en s'adressant aux Pharisiens ( $L$ uc 17,20). L'enracinement du moine en dialogue ne doit donc pas être compris comme un retour à la religion institutionnelle, soucieuse d'elle-même et occupée à séparer ce qui est orthodoxe et vrai de ce qui ne l'est pas; bien au contraire, le contact profond avec les autres croyants invite à faire sien le point de vue de Jésus qui prêche le R ègne, la conversion et l'amour des ennemis, plutôt qu'à se conformer à celui des instances ecclésiastiques qui prêchent la christologie et la théologie justes.

Le dialogue intrareligieux fait inévitablement éclater nos représentations de Dieu. H. Le Saux, B. Griffiths, J. Conner, J. W iseman ou encore T. Keating, soutiennent que le dialogue en profondeur est I'occasion de purifier le concept même de D ieu et d'éviter la tentation del'idolâtrie subtile qui consiste à concevoir Dieu à son image. Devant le

28. Voir le document audiovisuel sur B. Griffiths: The Space in the H eart of the Lotus, Orsino Productions, octobre 1987.

29. Voir Bulletin of M onastic Interreligious D ialogue 60 (1998), p. 9.

30. Ramana Maharshi est reconnu comme étant I'un des plus grands mystiques hindous de ce siècle.

31. Voir l'entrevue que j'ai faite avec 0 . Baumer-Despeigne, le 24 juillet 1996, à l'abbaye de G ethsémani (Kentucky).

32. "Contemplation et dialogue interreligieux. Repères et perspectives puisés dans l'expérience des moines », Bulletin. Secretarius pro non Christianis 84 (1993-X X VIII/3), p. 124. 
danger d'identifier la volonté de Dieu à celle de I'homme, D. SteindlRast applique à Dieu la fameuse sentence bouddhique : « si tu rencontres le Bouddha, tue-le ». De son côté, $R$. Panikkar affirme, au risque de choquer, que Dieu n'a pas de volonté. ${ }^{33}$ Le moine en dialogue institue le procès de Dieu contre Dieu et c'est en cela précisément que le dialogue est silencieux. Ici, le silence ne traduit pas un désengagement par rapport à sa foi et à ses convictions les plus profondes, mais renvoie à I'humilité qui grandit au fur et à mesure que le moine descend au cœur de l'expérience chrétienne, qui est essentiellement une expérience de mort-résurrection et de conversion ( $\mu \varepsilon \tau \alpha v o \iota \alpha)$. Le silence exprime à la fois le deuil de ce que R. Vachon a appelél' « ego chrétien » ${ }^{34}$ et la simplicité $(\alpha \pi \lambda \omega \sigma)$ de cœur. Sous l'effet de celle-ci, le moine se prosterne devant I'Indicible et s'émerveille face à la présence mystérieuse de D ieu dans le monde. $D$ 'où l'expression de son refus de monopoliser la vérité et sa volonté d'être à l'écoute de D ieu là où il fut longtemps interdit de le chercher : au cœur de l'altérité religieuse. Le moine en dialogue redécouvre ainsi l'importance de l'Esprit Saint à l'œuvre dans les différentes religions. H. Le Saux n'a t-il pas déclaré en effet : « $O$ Seigneur, je suis venu en Inde pour te faire connaître à mes frères hindous. Et c'est toi qui t'es fait connaître à moi à travers la méditation. Pourquoi t'es tu caché de cette façon pour me donner ta grâce? ${ }^{35}$ A insi, la différence religieuse n'est plus perçue comme une menace, mais bien comme une chance, celle de s'ouvrir à l'universalité de la révélation chrétienne, tout en reconnaissant le droit à l'auto-compréhension que son partenaire de dialogue a de lui-même en tant que croyant.

\subsection{L e silence du cheminement intérieur}

Enfin, troisième caractéristique, le dialogue des moines engage l'individu dans un processus de transformation intérieur, dont l'origine est à situer dans la coexistence délicate en soi de deux univers reli-

33. R. Panikkar écrit : « This assertion can shock but can also liberate us from the near divinization of the human will in western culture and philosophy. To silence the will is to go beyond the will. It is the work of grace and the outcome of purity of heart "; "John M ain Seminar Led by Panikkar », Bulletin of M onastic Interreligious Dialogue 57 (1997), p. 5.

34. Bulletin. N orth A merican Board for E ast-West Dialogue 3 (1978), p. 6. 35. P. CofF, «Abhishiktananda. An Interview with Odette BaumerDespeigne », Bulletin of M onastic I nterreligious D ialogue 51 (1994), p. 22. 
gieux. Si, dans I'hospitalité, l'autre devient un ami, il demeure malgré tout un étranger (hostis). Le dialogue est contraire à la tentative de s'identifier à l'autre, d'effacer les spécificités, parfois insurmontables, de chacun pour se réfugier dans un espèce de flou absolu qui s'imposerait à tout le monde, mais dans lequel personne ne saurait se reconnaître. Si les partenaires du dialogue sont déjà un, s'ils tendent vers un ultime commun, cela n'implique pas le rejet des divergences et des barrières qui les séparent. La rencontre en profondeur ne commande pas de partager ses sentiments les plus intimes, mais impose le respect des limites et des « zones de mystère » ${ }^{36}$ de chacun. Si les convergences sont nécessaires, parce qu'elles sont l'occasion d'un rapprochement et offrent un terrain commun propice à une rencontre authentique, les divergences sont toutes aussi déterminantes, car elles garantissent altérité, relation et tensions, sans quoi il ne peut y avoir ni recherche de la foi ${ }^{37}$, ni compréhension mutuelle. ${ }^{38}$

Encore une fois, il importe d'accueillir en soi l'expérience religieuse de l'autre dans ce qu'elle a de plus spécifique et d'universel. II faut pré server la cohérence des systèmes religieux de chacun, mais, dans certains cas, la coexistence de tels systèmes peut être source de tensions internes difficiles à gérer et de souffrances à la limite de l'insoutenable. Songeons à l'exemple d'H . Le Saux, qui rapporte que la présence de l'Évangile et des U panishads dans son cœur produisit un tel déchirement intérieur que seule la mort semblait en mesure de l'en délivrer. ${ }^{39}$ L'expérience intrareligieuse pousse en réal ité le moine à quitter un certain matérialisme spirituel pour retrouver sa condition d'exilé et de pèlerin. Désormais plus rien nesera comme avant, n'ayant nulle part où reposer la tête.

36. P.-F. DE BÉTHUNE, «L'Hospitalité religieuse», dans Par la foi et I'hospitalité, Clerlande, Publications de Saint-André (Cahier de Clerlande, 4), 1997, p. 32.

37. «Contemplation et dialogue interreligieux. Repères et perspectives puisés dans l'expérience des moines », Bulletin. Secretarius pro non Christianis 84 (1993-XX VIII/3), p.120.

38. R. Panikkar soutient que "la fécondation de deux traditions est possible en sacrifiant sa vie dans l'essai de soutenir les tensions existantes sans devenir schizophrène et de maintenir les polarités sans céder à une paranoïa personnelle et culturelle»; Bulletin. N orth A merican Board for East-West Dialogue 8 (1980), p. 2.

39. P. Coff, op.cit., p. 18.20 . 
Les repères et les murailles idéologiques qui donnaient I'apparence d'être à toute épreuve s'effondrent soudainement pour laisser place au vide, un vide de certitudes, de réponses et même d'arrogance. De ce vide surgit alors un silence, celui du questionnement, de l'angoisse et de l'instant présent, dont l'inconnu est désormais le seul horizon.

«Q Qui suis-je? » est la question cruciale que le moine est appelé très vite à se poser. Cette remise en question touche l'individu autant dans ce qu'il est en tant qu'être humain que dans son rapport à l'absolu. Peut-on encore défendre ici l'idée d'une dualité définitive entre créateur et créature à la manière de J. R atzinger dans sa Lettre de 1989 sur « Q uelques aspects de la méditation chrétienne »? Cela semble difficile pour ces moines qui, en s'engageant dans une pratique comme le yoga ou le zen, sont d'emblée confrontés au questionnement identitaire qu'H. Le Saux et B. $G$ riffiths ont hérité de $R$ amana $M$ aharshi. ${ }^{40}$ Pour le Bénédictin français, ce « qui suis-je? » renvoie au cœur de la spiritualité hindoue, à l'advaita. Dans le bouddhisme, cette question se rapporte à la notion de vacuité (sûnyatâ) et se comprend comme un moyen habile (upâya) d'utiliser la raison discursive pour éveiller les êtres à leur véritable nature, la nature de Bouddha, en réalisant que le « Je » ou le « moi » n'a pas d'existence en soi (anâtman). Par conséquent, l'expérience intrareligieuse pose nécessairement la question de l'identité chrétienne à partir d'une remise en cause radicale de l'anthropologie et de la vision du monde à travers lesquelles s'exprime la foi. Le moine en dialogue rend compte de son identité paradoxalement par la non-identité, en ce sens que le chrétien est celui qui meurt à lui-même et à tout ce qui fonde l'identité extérieure qu'il s'est forgée ${ }^{41}$ : « II n'y a pas de définition de la singularité chrétienne, écrit $C$. Geffré, en dehors de la croix du Christ comme figure de l'amour absolu. Ainsi, l'identité chrétienne appelle son propre dépassement ». ${ }^{42}$

40. Voir P. Coff, «Benedictine Spiritualtity and India's Religion», Spirituality Today 32/3 (septembre 1980), p. 239.

41. Voir H. Le SAUX, op.cit., p. 7. T. M erton écrit de son côté : « [...] I must also let go my hold upon myself, and not retain the semblance of a self which is an object or a « thing 》. I too must be no-thing. And when I am no-thing, I am in the All, and Christ lives in me »; Bulletin of M onastic Interreligious Dialogue 49 (1994), p. 21.

42. C. GefFRÉ, "Le Fondement théologique du dialogue interreligieux », dans R encontre, dialogue, annonce, Paris, Faculté de théologie et des sciences religieuses de l'Institut Catholique de Paris, 1995, p. 106. 
Cela m'amène tout naturellement à l'idée qu'en définitive les moines en dialogue partagent moins leurs croyances et leurs réponses que leurs questions et leur pauvreté ; si bien qu'on assiste à des échanges allant dans le sens d'un appauvrissement mutuel. Cela consiste à marcher ensemble dans la simplicité, en se débarrassant des lourdeurs des traditions, et à ce que, dans leurs interactions, le chrétien devienneun meilleur chrétien, le bouddhiste un meilleur bouddhiste et l'hindou un meilleur hindou. D e cette façon, I'appauvrissement mutuel contribue à la réalisation du royaume de Dieu compris comme l'unité dans la diversité. En somme, ce sont les dimensions kénotique et agapeique de l'exploration mutuelle des moines chrétiens et surtout bouddhistes qui sont désignées avec force par l'expression paradoxale de « dialogue de silence ».

\section{Conclusion}

Le dialogue des moines se situe aux frontières du silence à plusieurs égards, mais avant tout parce qu'il est actualisation et rappel de cette idée - partagée par les partenaires chrétiens, bouddhistes et hindous - que tous les êtres sont fondamentalement un, au-delà des différences culturelles et religieuses. Les moines entrent en dialogue précisément en vertu de cette connivence inaccessible à la raison, dont aucun mot, aucun concept, aucune formule ne peut rendre compte. Pas question alors de chercher le compromis en vue d'une unité idéale, comme cela est souvent le cas dans l'œcuménisme ; les moines entreprennent plutôt de se connaître sur la base de leur unité primordiale. En d'autres termes, il s'agit moins d'établir un accord de paix que d'anticiper le banquet eschatologique réunissant des convives venus autant de l'O rient que de l'O ccident ( $M$ atthieu 8,11). Par conséquent, les négociations font place aux retrouvailles, si bien que le dialogue n'est pas seulement envisagé comme un simple face-à-face, mais dans un rapport de «foi-à-foi », caractérisé par une démarche qui vise à faire sienne la vérité universelle de l'autre et qui s'effectue dans le secret du « cœur ». D'où la référence au silence comme dimension essentielle du dialogue des moines. Toute exploration de ce dernier est en effet vaine si elle ne tient pas compte de cette dimension, qui par ailleurs mérite d'être examinée plus à fond, puisqu'elle permet au dialogue de devenir une solution réelle aux problèmes posés à la société par le plural isme religieux contemporain. Lorsque la parole rejette le silence, elle fait obstacle à l'écoute, à l'humilité et à la remise en question ; elle s'érige en vérité universelle et devient le lieu de réfé- 
rence à partir duquel toute autre parole est jugée inférieure. À l'inverse, la parole mariée au silence retrouve sa juste mesure ; ni vaine ni insignifiante, elle n'acquiert de sens que dans son propre dépassement, orientée vers l'Indicible. Le silence se présente donc comme le garant d'un dialogue où l'autre croyant est respecté dans toute sa différence sans qu'il faille pour cela renier la sienne.

\section{RÉSUMÉ}

Le silence comme lieu de communion dans l'expérience du dialogue interreligieux monastique. L'article veut articuler la signification et la place du silence dans la dynamique dialogale qui se dégage de la rencontre entre des moines chrétiens et des moines non-chrétiens.

\section{ABSTRACT}

Silence as a place of communication in the context of experiencing interreligious monastic dialogue. The article aims at articulating the significance and place of silence within the dialogical dynamics which result from the meeting of Christian and non-Christian monks. 